\title{
Self-similarity in particle-laden flows at constant volume
}

\author{
Natalie Grunewald • Rachel Levy • \\ Matthew Mata - Thomas Ward . \\ Andrea L. Bertozzi
}

Received: 27 December 2008 / Accepted: 28 September 2009 / Published online: 16 October 2009

(C) The Author(s) 2009. This article is published with open access at Springerlink.com

\begin{abstract}
This paper deals with the evolution of a localized, constant-volume initial condition on an incline into a spreading descending thin-film solution. Clear fluids in this geometry are known to have a front position that moves according to a $t^{1 / 3}$ scaling law, based on similarity-solution analysis by Huppert (Nature 300:427-429, 1982). The same dynamics are investigated for particle-laden flow using a recently proposed lubrication model and physical experiments. The analysis includes the role of a precursor in the model. In the lubrication model, the height of the precursor significantly influences the position of the fluid front, independent of particles settling in the direction of flow. By comparing theory with experiments it is shown that the $t^{1 / 3}$ scaling law persists, to leading order, for particle-laden flows with particle settling. However, additional physics is needed in the existing lubrication models to quantitatively explain departures from clear-fluid self-similarity due to particle settling.
\end{abstract}

Keywords Gravity-driven film flow $\cdot$ Riemann problems $\cdot$ Sedimentation $\cdot$ Systems of conservation laws · Thin liquid films

\footnotetext{
N. Grunewald $(\varangle)$

Institut für Angewandte Mathematik, Universität Bonn, Wegelerstr. 10, 53115 Bonn, Germany

e-mail: grunewald@iam.uni-bonn.de

\section{R. Levy}

Department of Mathematics, Harvey Mudd College, 301 Platt Blvd., Claremont, CA 91711, USA

e-mail: levy@hmc.edu
}

M. Mata - A. L. Bertozzi

Department of Mathematics, University of California Los Angeles, 520 Portola Plaza, Los Angeles, CA 90095-1555, USA

M. Mata

e-mail: matthewmata@math.ucla.edu

\section{A. L. Bertozzi}

e-mail: bertozzi@math.ucla.edu

T. Ward

Department of Mechanical and Aerospace Engineering, North Carolina State University, Raleigh, NC 27695-7910, USA

e-mail: tward@ncsu.edu 


\section{Introduction}

The modeling of gravity-driven particle-laden flow is of interest in the context of geological phenomena such as mudslides, and industrial applications such as food science [1,2]. This paper compares the behavior of a constantmass particle-laden fluid flow down an incline with a pure fluid flow. We compare the analytical solutions of the pure fluid flow to both physical experiments and numerical simulations of the particle-laden fluid (PLF) flow, using a model proposed in $[3,4]$.

In [5] a simple scaling law is derived for the average front position $x(t) \sim C \cdot t^{1 / 3}$ in the case of clear fluids. Comparison to both the PLF lubrication model and to physical experiments suggests that the Huppert scaling law is still valid to leading order for particle-laden fluids with moderate particle concentrations in the range 30-55\%. In this range, the PLF still behaves fluid-like, and settling of the particles is present but does not dominate the large-scale dynamics. The effects of settling in the direction of the flow can be visually observed in the experiments as a particle-rich ridge at the leading edge of the PLF. We compare different settling functions in our model to analyze this effect numerically. We also note that the lubrication models with settling require a precursor; they are singular at vanishing precursor $[3,4]$. Thus it makes sense to compare the dynamics of the lubrication model with settling to an exact solution of the problem without settling and with precursor.

The paper is organized as follows: Sect. 2 reviews the PLF lubrication model to be used here for numerical simulations. Section 3 rederives the original Huppert solution with the addition of a precursor layer in the model. This necessitates a more careful assessment of shock dynamics not present in the original Huppert derivation. Section 4 presents experimental results with heavy particles and Sect. 5 presents numerical results of the lubrication model with settling and comparison to both the clear-fluid model and to experiments.

\section{A lubrication model for particle-laden flow}

The flow of a particle-laden fluid down an inclined plane has recently been modeled by a system of scalar conservation laws [3,6],

$\frac{\partial h}{\partial t}+\nabla \cdot\left(h v_{\text {tot }}\right)=0$

$\frac{\partial(\phi h)}{\partial t}+\nabla \cdot\left(\phi h v_{p}\right)=0$.

The total mass of the fluid is conserved in (1), where $h(t, x)$ is the height of the fluid-particle mixture at time $t$ and position $x$ (oriented down the substrate). We assume uniformity in the transverse direction. The total volume of particles $\phi h$ is conserved in (2), where $\phi$ is the volume fraction of particles in the fluid. The total velocity of the mixture $v_{\text {tot }}$ is a depth-average as well as a volume-average of the speeds of the fluid and the particles. The velocity of the particles $v_{p}$ consists of $v_{\text {tot }}$ and an extra term $\left[(1-\phi) v_{\text {rel }}\right]$ due to the sedimentation of the more dense particles in the fluid:

$v_{p}=v_{\text {tot }}+(1-\phi) v_{\text {rel }}$.

We assume that the volume fraction of particles $\phi$ is uniform in the direction perpendicular to the substrate. It is shown in [7] that this assumption can be improved to a distribution that is stationary in time which balances sedimentation and shear-induced migration. We refer to [7] for a precise discussion. The depth-average of the velocity of the fluid, $v_{\text {tot }}$ found in (1), can then be derived via standard techniques in lubrication theory for thin liquid films [6], which is valid for fluids with small Reynolds numbers and characteristic height much smaller than the characteristic length. The first-order term describes its dominant behavior as:

$v_{\text {tot }}=\frac{h^{2}}{3 \mu(\phi)} \rho(\phi) g_{\|}$.

While the derivation is standard, it should be noted that the fluid viscosity $\mu(\phi)$ and density $\rho(\phi)$ are both functions of the particle volume fraction. Following the modeling suggested in $[8,9]$, we model the viscosity with the empirically derived model 
$\mu(\phi)=\mu_{f}\left(1-\phi / \phi_{m}\right)^{-2}$

with dynamic fluid viscosity $\mu_{f}$. The random packing fraction of spheres $\phi_{m}$ is the maximum possible volume fraction of spheres in a fluid. We use $\phi_{m}=0.58$, which is our experimentally determined value [10]. This value is also used in the literature; see e.g. [11]. The density is a linear combination of the density of the fluid $\rho_{f}$ and the density of the particles $\rho_{p}$

$\rho(\phi)=\rho_{f}(1-\phi)+\rho_{p} \phi$.

The other constant in (3) is the component of gravitational acceleration $g_{\|}=|g| \sin \theta$, where $\theta$ is the inclination angle of the substrate.

The velocity $v_{p}$ in the second conservation law (2) requires a more explicit description, since the theory for particle-laden flow is still relatively novel and continues to present many open questions, especially for shear-driven flows. Recall that the lubrication approximation used to derive (3), employs a depth-averaged velocity. We also employ a depth-averaged model for $v_{\text {rel }}$, which we assume is a product of three factors:

$v_{\text {rel }}=v_{s} f(\phi) w(h)$.

The Stokes settling velocity $v_{s}$ is the speed at which a single particle with diameter $d$ will settle in a fluid of a given viscosity and density:

$v_{s}=\frac{2}{9} \frac{\left(\rho_{p}-\rho_{f}\right) g(d / 2)^{2}}{\mu_{f}}$.

The other factors account for phenomena that reduce the speed of a single particle: hindered settling from adjacent particles and slowing due to proximity of particles to the substrate; see also [12].

A classical model for hindered settling was proposed by Richardson and Zaki in [13] and Buscall et al. in [14]:

$f(\phi)=\left(1-\phi / \phi_{m}\right)^{\alpha}$

with $\phi_{m}=1$ and empirically determined exponent $\alpha=5.1$. Cook [6], modified the function to include the maximum packing fraction of particles $\left(\phi_{m}=0.67\right.$ in their case) to avoid singular shocks in solutions to the Riemann problem for (1) and (2) that occur when $\phi_{m}=1$. This form also ensures that sedimentation stops once the maximum concentration is reached. We will compare results for $\alpha=1$ and $\alpha=5$ to probe the effect of the exponent in the hindered settling function. This is particularly relevant when comparing numerical results and physical experiments, since the division by the maximum packing fraction may have altered the appropriate choice of exponent for comparison to experiments. Note that, although the singular limits for both functions

$\lim _{\phi \rightarrow \phi_{m}} f(\phi)=0, \quad \lim _{\phi \rightarrow 0} f(\phi)=1$,

are appropriate, we will not consider extreme values of $\phi$, since the comparison to experiments is most appropriate for moderate concentrations of particles.

The third factor in (5) models the slowing of particles due to their proximity to the substrate, sometimes called the wall effect [4] and [6]

$w(h)=\frac{\frac{1}{18}(h / d)^{2}}{\sqrt{1+\left[\frac{1}{18}(h / d)^{2}\right]^{2}}}$.

Note that $w(h)$ is close to 0 for $h \ll d$ and close to 1 for $h \gg d$. The full system of equations for $h(t, x)$ and $\phi(t, x)$ is now fully specified by incorporating (3) into (1) and (5) into (2).

As has been shown for clear fluids [15], a first-order model such as the one proposed here can correctly capture quantities such as front speed but does not contain the physics necessary to model fingering due to surface tension and the component of gravity normal to the substrate. Numerical evidence in [6] for constant-flux boundary conditions indicates that higher-order terms smooth solutions but do not affect the speed of the leading front of the film. Nevertheless, quite a lot of information can be gained by studying the dominant physics in the flow direction, in particular with regard to the competition between settling of particles and overall motion of the fluid. 
Nondimensionalizing the reduced system with length scale $h_{0}$ (half of the upstream gate height) and timescale $t_{0}=\sin \theta t$, we obtain

$$
\begin{aligned}
& \partial_{t} h+\partial_{x}\left(\frac{g \rho(\phi)}{3 \mu(\phi)} h^{3}\right)=0, \\
& \partial_{t}(h \phi)+\partial_{x}\left(\frac{g \rho(\phi)}{3 \mu(\phi)} \phi h^{3}+v_{s} \phi h(1-\phi) f(\phi) w(h)\right)=0,
\end{aligned}
$$

which we solve numerically and compare to experimental results. Note that this system has been studied in [6] for constant-flux boundary conditions. Since the physical experiment (described in Sect. 4) more closely resembles a constant volume of PLF than a constant flux (for the purpose of numerical simulations), we will choose initial conditions to approximate a constant volume of fluid.

\section{Well-mixed similarity theory including precursor}

We begin our discussion of similarity solutions by considering a model for a well-mixed fluid-particle flow down an incline. Let $\phi_{0}$ be the initially constant volume fraction of particles in the fluid. For a well-mixed fluid without particle settling the volume fraction stays constant in space and time. We compare the settling model (7) and (8) to solutions of the well-mixed model:

$h_{t}+\frac{g \rho_{0}}{3 \mu_{0}}\left(h^{3}\right)_{x}=0$

with

$\rho_{0}=\rho\left(\phi_{0}\right) \quad$ and $\quad \mu_{0}=\mu\left(\phi_{0}\right)$.

This model from [5] captures the dominant behavior of the flow with no particles. For simulations with no precursor, the solution of (9) for an initial fluid profile

$h_{0}(x)= \begin{cases}\beta & \text { if } x \in[0, a] \\ 0 & \text { else }\end{cases}$

can be solved explicitly: We consider (9) in its natural timescale

$\tau=\frac{g \rho_{0}}{\mu_{0}} t$

After an initial transient time $\tau \geq \tau^{*}=\frac{3 a}{2 \beta^{2}}$ a similarity solution

$h(\tau, x)= \begin{cases}\sqrt{x / \tau} & \text { if } x \in\left[0, x_{s}(\tau)\right] \\ 0 & \text { else }\end{cases}$

develops with

$x_{s}(\tau)=\left(\frac{3}{2} \beta a\right)^{2 / 3} \tau^{1 / 3}=C \cdot t^{1 / 3}$,

where

$C=\left(\frac{3}{2} \beta a\right)^{2 / 3}\left(\frac{g \rho_{0}}{\mu_{0}}\right)^{1 / 3}$.

The constant $C$, when measured experimentally, provides a method for measuring the effective viscosity of the fluid by inverting (11)

$\mu=\frac{9}{4} \beta^{2} a^{2} g \rho_{0} C^{-3}$. 
The effectiveness of this quantity as a measure for bulk viscosity of the PLF is explored in [10]. For comparison between numerical experiments and simulations, we focus on the variation in the scaling constant $C$ for experiments with and without particles.

It is also possible to consider analytical solutions of (9) with a small uniform precursor layer of height $b$ : We consider (9) with Riemann initial data

$h_{0}(x)= \begin{cases}\beta & \text { if } x \in[0, a] \\ b & \text { else. }\end{cases}$

The discontinuity in the initial data at $x=0$ will immediately become a rarefaction, while the shock at $x=a$ will persist. At a critical time

$\tau_{c}=\frac{a}{\beta^{2}-\frac{1}{3}\left(\beta^{2}+\beta b+b^{2}\right)}$,

the trailing rarefaction and leading shock will merge, creating a wedge shape that continues to evolve in time above the precursor. The shape of this profile can be described analytically [16,17]. Above the precursor the solution still has the shape of $\sqrt{x / \tau}$. The excess volume above the precursor

$V=a(\beta-b)$

remains constant. One can therefore use the conservation of mass to determine the speed of the shock. Let $x_{s}$ be the position of the shock. Then

$\int_{b^{2} \tau}^{x_{s}} \sqrt{x / \tau} \mathrm{d} x-b\left(x_{s}-b^{2} \tau\right)=V$.

Therefore the shock position is defined implicitly by the solution of

$\frac{2}{3}\left(x_{s} \sqrt{x_{s} / \tau}-b^{3} \tau\right)-b\left(x_{s}-b^{2} \tau\right)-V=0$

and the solution of the double Riemann problem with precursor after $\tau_{c}$ is

$h(\tau, x)= \begin{cases}b & x<b^{2} \tau, \\ \sqrt{x / \tau} & b^{2} \tau<x<x_{s}, \\ b & x>x_{s} .\end{cases}$

To explore the difference in evolution of the solutions analytically, let

$H=\sqrt{\frac{x_{s}}{\tau}}$

be the maximum height of $h$. Rewriting (13) in $V$ and $H$ gives:

$\frac{2}{3} H^{3}-V / \tau=b H^{2}-b^{3} / 3$.

This illustrates by (15) and (12) that, as long as $b \ll H$, the right-hand side is negligible and $x_{s}$ approximates the shock position of the solution without precursor (10). Figure 1 shows the deviation of the shock positions. The data start from time $\tau_{c}$ after which (13) is valid. For increasing time and precursor the relation $b \ll H$ gets less valid and therefore the deviation of the shock positions increases. Note that for the settling model the limit $b \rightarrow 0$ is singular $[4,3]$. We therefore have to introduce a precursor for the numerical simulations.

\section{Experimental results}

The experimental apparatus consists of a $100 \mathrm{~cm}$ long, $50 \mathrm{~cm}$ wide acrylic sheet mounted to an adjustable stand capable of inclination angles ranging from $5^{\circ}$ to $80^{\circ}$. Down the length of the substrate is a track approximately 
Fig. 1 Comparison of front position for different precursor heights, at $a=2.5, \beta=2$ and $\phi=0.4$

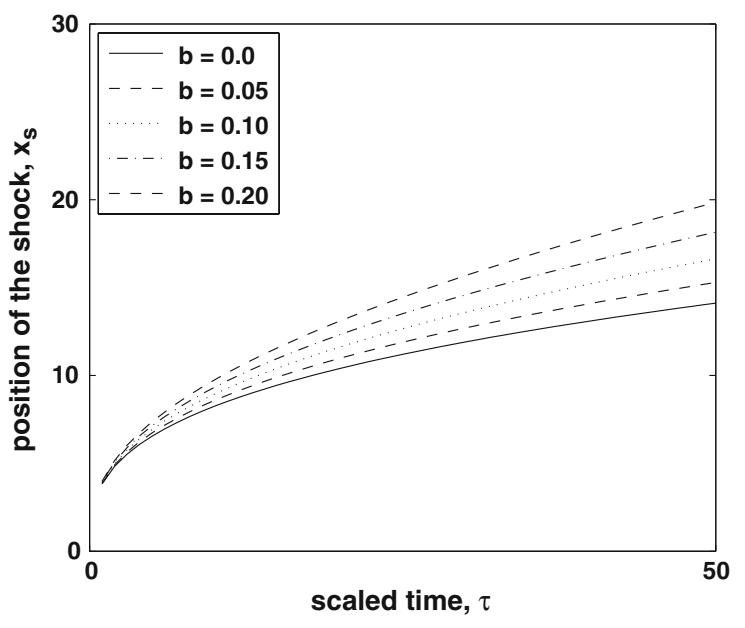

$14 \mathrm{~cm}$ wide. Side walls, approximately $3.2 \mathrm{~cm}$ high near the top and $1.4 \mathrm{~cm}$ near the lower part of the track are designed so the fluid does not escape over the sides. Near the top of the acrylic sheet is a gated reservoir from which a finite well-mixed volume of fluid and particles is released.

The experiments shown are all conducted at an inclination angle of $45^{\circ}$ and particle concentrations of $25-45 \%$, in increments of $5 \%$. For these values we avoid the rapid settling of the particles toward the substrate associated with low concentrations and low inclination angles which leads to deposits of particles at the rear of the flow and clear fluid fingers at the leading edge. We also avoid particle jamming, clumping and sliding that is associated with higher particle concentrations and higher inclination angles. Additional experimental data have been collected for other inclination angles [10]; but the data presented here for $45^{\circ}$ provide representative results.

The PLF solution is a mixture of $1,000 \mathrm{cSt}$ silicone oil (Clearco Products) with a density of approximately $0.96 \mathrm{~g} / \mathrm{cm}^{3}$, and soda-lime glass beads (Ceroglass) with a density of approximately $2.5 \mathrm{~g} / \mathrm{cm}^{3}$. The diameter of the beads is $0.025 \mathrm{~cm}$. For smaller particles, the settling in the direction of the flow to form a particle ridge at the front is less evident.

This experimental setup has a Peclet number Pe on the order of $10^{10}$, with $\mathrm{Pe}=v_{s} d / 2 D$ where $v_{s}=2\left(\rho_{p}-\rho_{f}\right)$ $g(d / 2)^{2} /\left(9 \mu_{f}\right)$ is the settling velocity, $d$ is the particle diameter and $D=k_{b} T / 6 \pi \mu_{f}$ the diffusivity with Boltzmann constant $k_{b}$ and temperature $T$. We note that a high Peclet number in the experiments suggests that we may neglect Brownian motion in the derivation of the model.

The maximum packing fraction of beads is determined experimentally as described in [10]. The value of $\phi_{m}$ is measured to be approximately $0.57-0.58$. Each preparation of PLF has a constant volume of $90 \mathrm{~cm}^{3}$ with approximately $70 \mathrm{~cm}^{3}$ actually being transferred from the jar into the reservoir. The particle-fluid mixture is prepared according to the desired volume fraction of beads and silicone oil.

To begin the experiment, the PLF materials are placed in a plastic container and hand-mixed using a stirring rod for $4 \mathrm{~min}$, creating a homogeneous mixture. Since the density of the particles is greater than that of the fluid, the particles settle out fairly quickly and the experiments must be performed immediately after the PLF is well-mixed. The particle-laden fluid is placed in the reservoir and the gate is opened. A camera positioned above the track and perpendicular to the substrate records still images at predetermined time intervals. The images for the 25 and $30 \%$ particle volume fraction experiments are recorded at $4 \mathrm{fps}$ (frames per second), the 35 and $40 \%$ at $2 \mathrm{fps}$, and the $45 \%$ at $1 \mathrm{fps}$.

The images are analyzed by an image-processing code and an average front position is calculated for each image. Figure 2 shows a time-series of images taken at $45 \mathrm{~s}$ intervals. In these images the development of the fingering instability and a dark particle-rich ridge at the front of the PLF can be observed. Figure 3 contains a series of plots each taken $2 \mathrm{~min}$ into the experiment for a range of particle volume fractions. Note that the slurries with more particles move more slowly, and have a darker particle ridge at the front. 

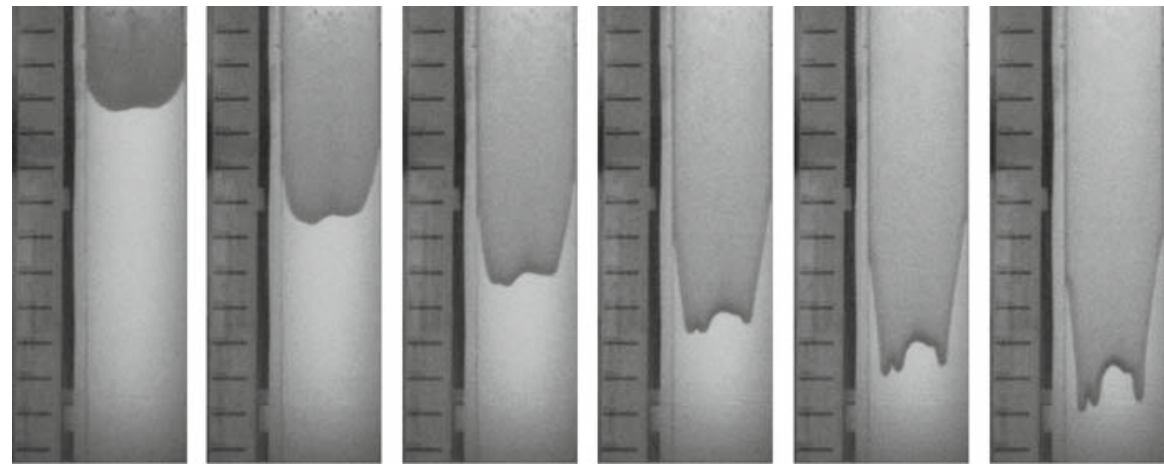

Fig. 2 Time series $45 \mathrm{~s}$ apart, 35\% particle volume fraction, $45^{\circ}$ inclination
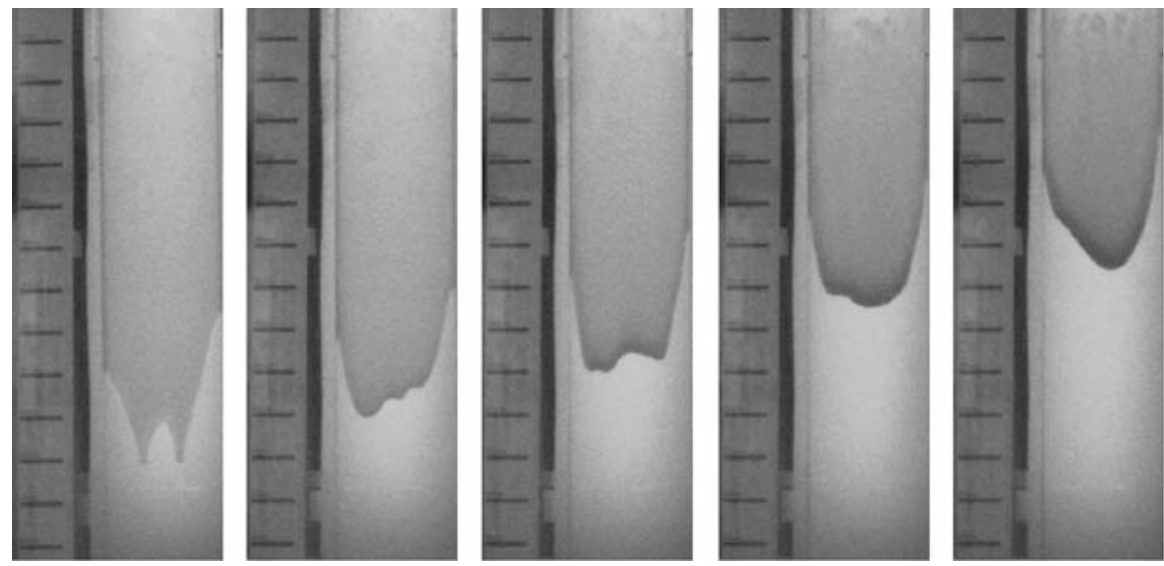

Fig. 3 Varying particle volume fractions 25, 30, 35, 40 and $45 \%$ at $2 \mathrm{~min}$

Fig. 4 Experimental results tracking the average front position and plotting it against the rescaled time $\tau^{1 / 3}$

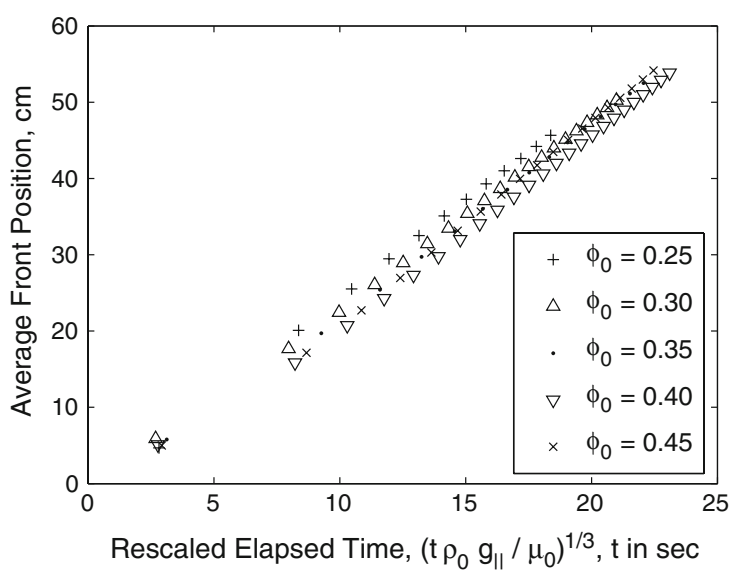

The images recorded by the camera are processed to extract the profile of the leading edge of the fluid. A front position for the central half of the flow (away from the side walls), measured in pixels and averaged over approximately 125 data points, is calculated and later converted into a physical distance. The average front position $(\mathrm{cm})$ is plotted against the rescaled time $\tau$ (see Sect. 3) to the one-third power in Fig. 4. After an initial transient, the data is approximately linear with slope (found using least squares) analogous to the scaling constant $C$ of (11), which decreases with increasing particle concentration. This qualitatively confirms the well-mixed model. A quantitative 

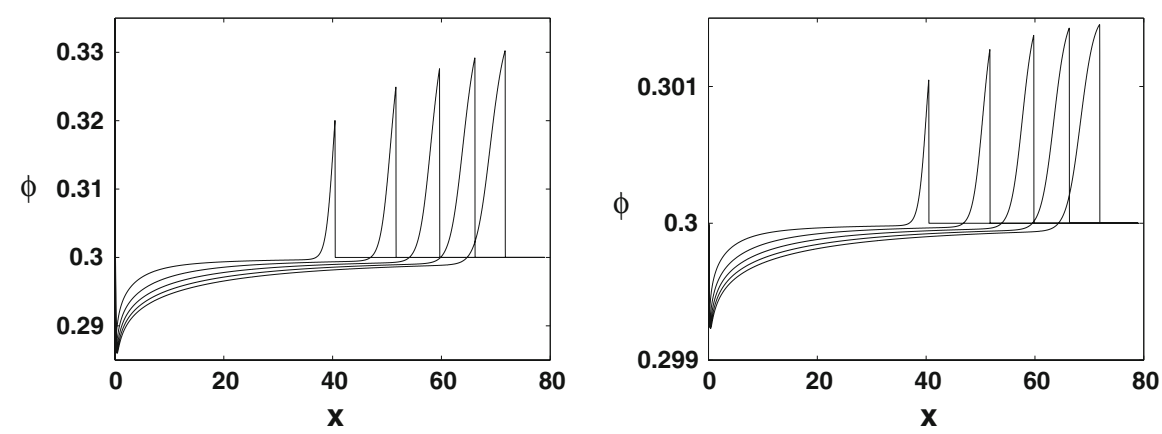

Fig. 5 Numerical simulations of the particle concentration of solutions of (7) and (8) with initial concentration $\phi_{0}=0.3$, precursor height 0.01 and for settling function (6) with $\alpha=1$ (left) and $\alpha=5$ (right) at times $t=30,60,90,120$. While the plots look qualitatively similar, the more singular solutions for $\alpha=1$ have larger maxima

comparison of the theoretical, experimental and numerical scaling constants is given in Fig. 9. Note also that the data for the different particle volume fractions almost collapse.

\section{Numerical simulations}

In this section we describe numerical simulations for the model of a particle-laden film with settling, i.e., (7) and (8). This model requires a precursor as it has been shown that solutions with settling depend singularly on the precursor thickness [3,4]. The simulations employ an upwind finite-difference scheme, which is efficient for conservation laws with a unidirectional velocity. We use the experimental parameters described in Sect. 4 for the viscosity, density and the diameter of the particles. For the maximum packing fraction $\phi_{m}$ we use the experimentally determined value of 0.58; see [10]. We assume initial data of the step-like form described in Sect. 3, with initial height $\beta=2$ and width $a=2.5$. The precursor height for the numerical data is $b=0.01$ unless otherwise stated. The initial particle concentration is uniform, representing the well-mixed initial PLF in the physical experiment.

We compute solutions with settling function (6) for $\alpha=1$ and $\alpha=5$. Recall that the value for the exponent $\alpha$ that was determined experimentally [13]. For each of the settling functions, Fig. 5 contains snapshots in time of numerical solutions of (8). In the left-hand plot which contains solutions using the settling function (6) with $\alpha=1$, the magnitude of the plots in the vertical direction is larger than solutions using the settling function (6) with $\alpha=5$. This phenomenon has been described in [3] as a singular shock. However, at these scales, the plots are qualitatively similar. From the modeling point of view the exponent $\alpha=1$ seems to be more appropriate as it shows a stronger ridge that is also observed experimentally. For $\alpha=5$ the concentrations vary less than 1\%. From the data in Fig. 5 one can determine the spacial variations in the viscosity by the empirically derived model (4).

In Fig. 6, we compare the profile shapes for the analytical solution with precursor (left) and the numerical solution with settling function (6). To compare profile shapes we rescale the data by maximum height in $h$ and by front position in $x$. We see that the addition of particle settling exhibits departures from pure self-similarity, as seen in the profile shapes, for times relevant to us. Another comparison of the fronts is illustrated in Fig. 7. It shows that the height of the precursor makes a much more significant difference in the front speed than the addition of particle settling. With a precursor, the front speed is similar for both the analytical solution and the settling models of either power and faster than that of the similarity solution with no precursor.

Figure 8 is the numerical version of Fig. 4 in the physical experiments. It shows the same near-collapse of the front positions in the right scaling. The data is ordered monotone with the particle concentration. The qualitative agreement of Figs. 4 and 8 is very good, whereas the quantitative agreement is not yet fully reached.

A quantitative comparison between analytical, numerical and experimental results is provided in Fig. 9. It shows the scaling constants derived from the experiments, the numerics and the theory, all divided by the theoretical constants, in dependence of the particle volume fraction. The solid line at 1 represents the theoretical constants (11) 

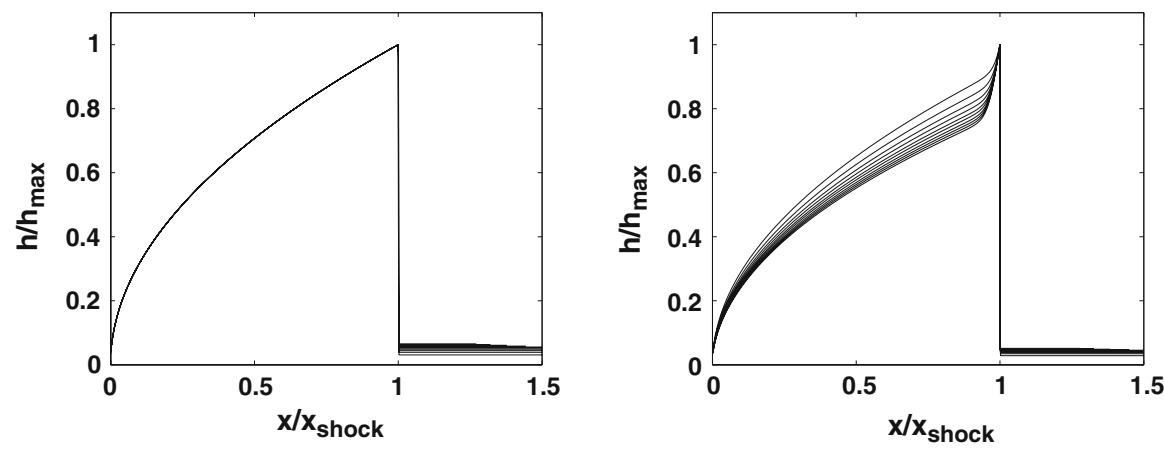

Fig. 6 A comparison of scaled solutions. On the left, simulations of (7-8) with precursor but no particle settling. On the right, simulations with precursor and settling with $\alpha=1$. Both simulations have precursor height 0.05 and initial concentration $\phi=0.4$

Fig. 7 Comparison of solutions to the settling model (7-8) with analytical solutions (14) of the Riemann problem with precursor height $b=0.01$ and without precursor for initial volume fraction of particles $\phi=0.3$, at time $t=30$

Fig. 8 The front position $x_{S}(\tau)$ plotted versus $\tau^{1 / 3}$ for different particle volume fractions. The data is nearly independent of the choice of settling function. It has precursor height $b=0.01$
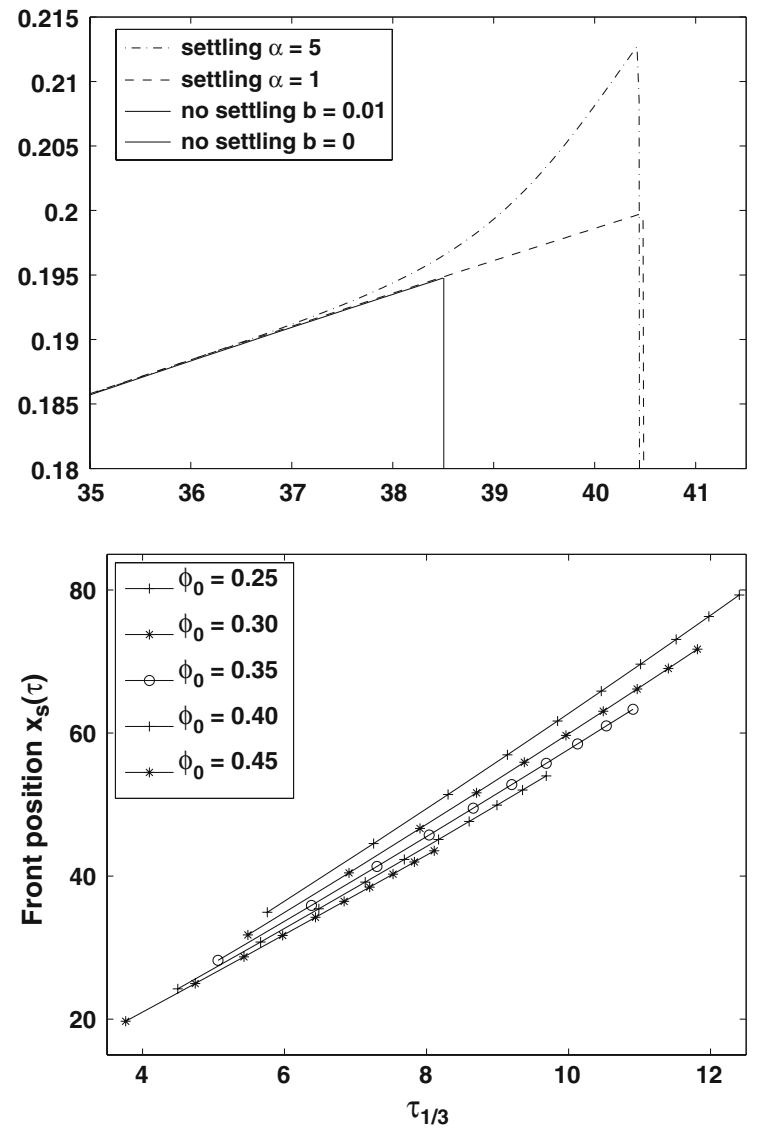

derived from the analytical solutions without precursor. The theoretical constants with precursor height $b=0.01$ derived in Sect. 3 are bigger than the ones for $b=0$. They are similar to the numerical data for precursor height $b=0.01$. The numerical values for $C$ for the different settling functions are so similar that the points are virtually indistinguishable on this plot and are represented by a single averaged point. The numerical data is the same as in Fig. 8. The numerical (experimental) constants are found by the slope of a linear interpolation of the data in Fig. 8 (Fig. 4). The best agreement between the numerical results from the system (7) and (8) and the experimental results is for an initial particle concentration of approximately 0.45 . We conjecture that this is a balancing point between low particle concentrations when settling to the substrate is dominant and high particle concentrations 
Fig. 9 Dependence of the scaling constant $C$ on the particle concentration $\phi$. The theoretical scaling constant is computed from (11). The numerical and experimental data come from Figs. 8 and 4

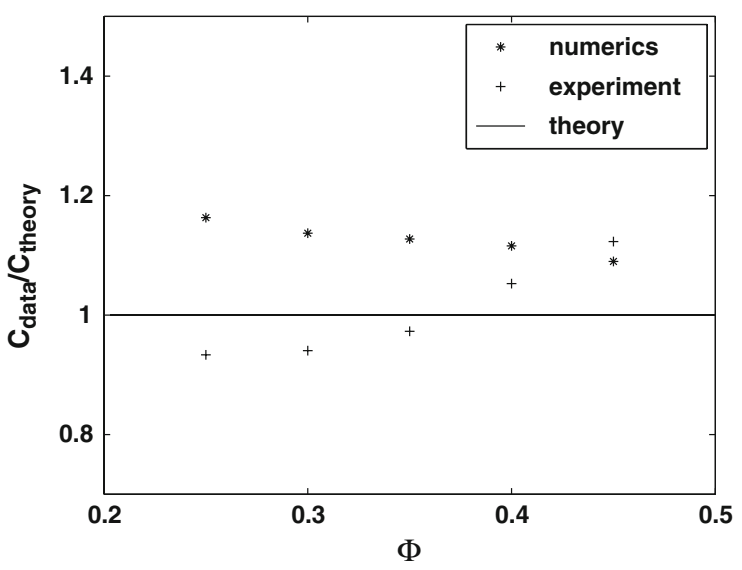

when clumping and sliding behavior dominates. We see that numerics as well as theory overestimates the scaling constant found in the physical experiment for low concentration. For high concentrations the numerical and the experimental scaling constants almost agree.

\section{Conclusion}

We have developed a new understanding of the role of the precursor in lubrication models for thin-film slurries, in determining the position of the front of a particle-laden fluid. Settling of the particles in the direction of the flow primarily affects the profile of the film as a particle-rich ridge develops at the front, and has a less significant effect on the speed of the front. The scaling for the front position that is exact for analytical solutions with and without precursor is also appropriate to leading order for numerical simulations of the model with particle settling, as well as for the physical experiments.

The development of a particle rich ridge at the front of the PLF is seen in the numerical data and in the physical experiments, where it appears as a dark ridge at the front. However, it is also clear that the model does not quantitatively reproduce this departure from self-similarity of the fluid profile. A separate paper, on the transverse fingering instability with surface tension [18] also suggests that some important physics is missing from the lubrication model, even when surface-tension effects are included in the mathematics. One possibility of additional physics to include in this analysis is shear induced migration (e.g. [19,20]) which has recently been shown to give quantitatively accurate predictions of phase transitions between settling to the substrate and settling to contact line in constant flow-rate experiments [7]. It would also be interesting to incorporate a precursor into the physical experiments.

The understanding of particle-laden fluid flows and the relationship to pure fluid and pure granular models is still at a preliminary stage. Appropriate modeling for low concentrations, in which settling to the substrate creates a phase transition in which part of the fluid becomes particle-free needs more modeling. The high particle concentrations that exhibit sliding of large clumps will require yet another (if fluid, then non-Newtonian) model.

Acknowledgement Natalie Grunewald was supported by the DFG grant GR 3391/1-1. She thanks Andrea Bertozzi and UCLA for their kind hospitality during the last year. This work was supported by ONR grant N000140610059, and NSF grants ACI-0321917, DMS-0601395, and DMS-0502315, as well as UC Lab Fees Research Grant number 09-LR-04-116741-BERA. We thank Ben Cook and A. E. Hosoi for helpful discussions.

Open Access This article is distributed under the terms of the Creative Commons Attribution Noncommercial License which permits any noncommercial use, distribution, and reproduction in any medium, provided the original author(s) and source are credited. 


\section{References}

1. Monquet C, Greffeuille V, Treche S (2006) Characterization of the consistency of gruels consumed by infants in developing countries: assessment of the Bostwick consistometer and comparison with viscosity measurements and sensory perception. Int $\mathrm{J}$ Food Sci Nutr 57:459-469

2. Rao R, Mondy L, Sun A, Altobelli S (2002) A numerical and experimental study of batch sedimentation and viscous resuspension. Int J Numer Methods Fluids 39:465-483

3. Cook BP, Bertozzi AL, Hosoi AE (2007/2008) Shock solutions for particle-laden thin films. SIAM J Appl Math 68(3):160-183

4. Zhou J, Dupuy B, Bertozzi AL, Hosoi AE (2005) Theory for shock dynamics in particle-laden thin films. Phys Rev Lett $94: 117803$

5. Huppert HE (1982) Flow and instability of a viscous current down a slope. Nature 300:427-429

6. Cook BP (2007) Lubrication models for particle-laden thin films. PhD thesis, UCLA CAM report (07-42)

7. Cook BP (2008) Theory for particle settling and shear-induced migration in thin-film liquid flow. Phys Rev E 78:045303

8. Krieger IM (1972) Rheology of monodisperse lattices. Adv Colloid Interface Sci 3:111-136

9. Stickel JJ, Powell RL (2005) Fluid mechanics and rheology of dense suspersions. Annu Rev Fluid Mech 37:129-149

10. Ward T, Wey C, Glidden R, Hosoi AE, Bertozzi AL (2009) Experimental study of gravitation effects on the effective shear viscosity of a particle-laden thin film on an inclined plane. Phys Fluids 21:083305

11. Zarraga IE, Hill DA, Leighton DT Jr (2000) The characterization of the total stress of concentrated suspensions of noncolloidal spheres in Newtonian fluids. J Rheol 44:2

12. Taylor GI. Sedimentation and falling bodies: low Reynolds number flow. Film, available from http://web.mit.edu/fluids/www/ Shapiro/ncfmf.html

13. Richardson JF, Zaki WN (1954) Sedimentation and fluidization: part I. Trans Inst Chem Eng 32:35-53

14. Buscall B, Goodwin JW, Ottewill RH, Tadros TF (1982) The settling of particles through Newtonian and non-Newtonian media. J Colloid Interface Sci 85:78-86

15. Bertozzi AL, Brenner M (1997) Linear stability and transient growth in driven contact lines. Phys Fluids 9(3):530-539

16. Lax PD (1973) Hyperbolic systems of conservation laws and mathematical theory of shock waves. In: CBMS-NSF regional conference series in applied mathematics, SIAM, vol 11

17. Evans LC (1998) Partial differential equations. American Mathematical Society, Providence

18. Cook B, Alexandrov O, Bertozzi A (2009) Linear stability and nonlinear dynamics in particle-laden thin films. Eur Phys $\mathrm{J}$ (to appear)

19. Leighton D, Acrivos A (1986) Viscous resuspension. Chem Eng Sci 41(6):1377-1384

20. Leighton D, Acrivos A (1987) The shear-induced migration of particles in concentrated suspensions. J Fluid Mech 181:415-439 